\title{
First Trimester
}

National Cancer Institute

\section{Source}

National Cancer Institute. First Trimester. NCI Thesaurus. Code C92799.

The period of gestation that ranges from the first day of the last menstrual period in a woman up to the 12 th week of her pregnancy. 